\title{
Approximated convex envelope of a function
}

Brighi, B ; Chipot, M

\begin{abstract}
The goal of this paper is to introduce the approximated convex envelope of a function and to estimate how it differs from its convex envelope. Such a problem arises in various physical situations where the function considered is some energy that has to be minimized.This study is a first step toward understanding how to approximate the quasi-convex envelope of a function. The importance of this issue is due to the various applications that are encountered, in particular, in the field of material science. (C)1994 (Copyright) Society for Industrial and Applied Mathematics
\end{abstract}

DOI: https://doi.org/10.1137/0731007

Posted at the Zurich Open Repository and Archive, University of Zurich ZORA URL: https://doi.org/10.5167/uzh-22621

Journal Article

Originally published at:

Brighi, B; Chipot, M (1994). Approximated convex envelope of a function. SIAM Journal on Numerical Analysis, 31(1):128-148.

DOI: https://doi.org/10.1137/0731007 


\title{
APPROXIMATED CONVEX ENVELOPE OF A FUNCTION*
}

\author{
BERNARD BRIGHI ${ }^{\dagger}$ AND MICHEL CHIPOT ${ }^{\dagger}$
}

\begin{abstract}
The goal of this paper is to introduce the approximated convex envelope of a function and to estimate how it differs from its convex envelope. Such a problem arises in various physical situations where the function considered is some energy that has to be minimized.

This study is a first step toward understanding how to approximate the quasi-convex envelope of a function. The importance of this issue is due to the various applications that are encountered, in particular, in the field of material science.
\end{abstract}

Key words. nonconvex energy, convex envelope, finite elements, approximation

AMS subject classifications. 26B25, 49XX, 52A20, 65N30, 65 N99

1. Introduction. In many physical problems - for instance in material science - one is lead to minimize nonconvex energies. The typical case is when a material has several natural states. Then one way to model the situation is to introduce an energy density with several minima, each of which is an account for this preferred state. Of course, such an energy with several "wells" is not convex. The energy level corresponding to a linear boundary deformation is the so-called quasi-convexification of the energy density - a mathematical concept introduced by C.B. Morrey (see [9]). This is an extremely useful quantity from both the theoretical point of view and the practical one. One of our goals is to analyze how accurate a finite element method is to approximate it (see [1]). A first step toward this is to understand the scalar case (i.e., in mechanics the deformation is a vector but here we analyze the case where our unknown is a scalar (see [9])). Then in this case the quasi-convexification is simply the convexification of the energy density, i.e., the largest convex function bounding this density from below. In this paper we would like to investigate how it can be approximated by a $P_{1}$-finite element method. One could think to use our analysis to compute the convex envelope of some domains - for instance, the epigraph of the convexification of a function is the closed convex envelope of the epigraph of this function. We did not pursue this analysis here, however.

Consider $n>0$ as an integer and $\Omega$ as a bounded domain of $\mathbb{R}^{n}$ assumed to be polygonal for the simplicity of the numerical analysis. Let

$$
\varphi: \mathbb{R}^{n} \rightarrow \mathbb{R}
$$

be a function bounded from below by an affine function, so that there exists

$$
g: \mathbb{R}^{n} \rightarrow \mathbb{R} \text { affine such that } g \leq \varphi
$$

Let us denote by $\varphi^{* *}$ the convex envelope of $\varphi$, i.e., the function

$$
\varphi^{* *}=\sup \left\{\psi: \mathbb{R}^{n} \rightarrow \mathbb{R}: \psi \text { convex and } \psi \leq \varphi\right\}
$$
1993.

*Received by the editors March 20, 1992; accepted for publication (in revised form) March 1,

†Université de Metz, U.F.R. "M.I.M." Département de Mathématiques et Informatique, Ile du Saulcy, 57045 Metz Cedex 01, France. 
By (1.1) we see that $\varphi^{* *}$ is finite. Moreover, we have

$$
\forall \alpha \in \mathbb{R}^{n}, \quad \varphi^{* *}(\alpha)=\operatorname{Inf}_{v \in W_{0}^{1, \infty}(\Omega)} \frac{1}{|\Omega|} \int_{\Omega} \varphi(\alpha+\nabla v(x)) d x
$$

In the above formula and below, we denote by $W^{1, \infty}(\Omega)$ the space of Lipschitz functions defined in $\Omega$ with values in $\mathbb{R}$; see [11]. $W_{0}^{1, \infty}(\Omega)$ is the subspace of functions of $W^{1, \infty}(\Omega)$ vanishing on $\partial \Omega$, the boundary of $\Omega .|\Omega|$ is the measure of $\Omega$. It is well known that the right-hand side of (1.2) does not depend on $\Omega$; see, for instance, [9].

Then let $\left\{\mathcal{T}_{h}: h>0\right\}$ be a family of regular triangulation of $\Omega$ (see [14]), that is to say, satisfying

$$
\forall h>0\left\{\begin{array}{l}
\forall K \in \mathcal{T}_{h}, \quad K \text { is a } n \text {-simplex, } \\
\max _{K \in \mathcal{T}_{h}}\left(h_{K}\right)=h, \\
\forall K \in \mathcal{T}_{h}, \quad \frac{h_{K}}{\rho_{K}} \leq \nu \quad(\nu>0),
\end{array}\right.
$$

where $h_{K}$ is the diameter of the $n$-simplex $K$ and $\rho_{K}$ is its roundness (i.e., the largest diameter of the balls that could fit in $K$ ).

If $P_{1}(K)$ is the space of polynomials of degree 1 on $K$, we set

$$
V_{h}^{0}=\left\{v: \Omega \rightarrow \mathbb{R} \text { continuous : }\left.v\right|_{K} \in P_{1}(K) \forall K \in \mathcal{T}_{h} \text { and } v=0 \text { on } \partial \Omega\right\}
$$

and, for $\alpha \in \mathbb{R}^{n}$,

$$
\varphi_{h}^{* *}(\alpha)=\operatorname{Inf}_{v \in V_{h}^{0}} \frac{1}{|\Omega|} \int_{\Omega} \varphi(\alpha+\nabla v(x)) d x
$$

One clearly has

$$
\varphi^{* *} \leq \varphi_{h}^{* *} \leq \varphi .
$$

We would like to derive estimates of $\varphi_{h}^{* *}-\varphi^{* *}$ here. Previous results of this type were obtained in the context of hyperelasticity for ordered materials (see [4]-[8]). However, in all these papers the functional $\varphi_{h}^{* *}$ did not emerge clearly. We will call it the approximated convex envelope of $\varphi . \varphi_{h}^{* *}$ depends, of course, on $\Omega$, but for simplicity we will drop this dependence, $\Omega$ being fixed. In physical situations, $\varphi$ is some energy that one tries to minimize.

Remark 1.1. For all $\alpha \in \mathbb{R}^{n}$, and for all affine functions $g: \mathbb{R}^{n} \rightarrow \mathbb{R}$, one has

$$
\begin{aligned}
(\varphi-g)_{h}^{* *}(\alpha) & =\operatorname{Inf}_{v \in V_{h}^{0}} \frac{1}{|\Omega|} \int_{\Omega}(\varphi-g)(\alpha+\nabla v(x)) d x \\
& =\operatorname{Inf}_{v \in V_{h}^{0}}\left[\frac{1}{|\Omega|} \int_{\Omega} \varphi(\alpha+\nabla v(x)) d x-g\left(\frac{1}{|\Omega|} \int_{\Omega}(\alpha+\nabla v(x)) d x\right)\right] \\
& =\operatorname{Inf}_{v \in V_{h}^{0}}\left[\frac{1}{|\Omega|} \int_{\Omega} \varphi(\alpha+\nabla v(x)) d x-g(\alpha)\right] \\
& =\varphi_{h}^{* *}(\alpha)-g(\alpha) .
\end{aligned}
$$


Therefore,

$$
(\varphi-g)_{h}^{* *}=\varphi_{h}^{* *}-g .
$$

2. A general convergence theorem. We will need the following lemma.

LEMMA 2.1. Let $v \in W^{1, \infty}(\Omega)$ and $v_{h}$ be its interpolate on the triangulation $\mathcal{T}_{h}$. Then there exists a constant $c \geq 1$ depending on $n$ and $\nu$ such that

$$
\left|\nabla v_{h}(x)\right| \leq c|| \nabla v \|_{\infty, \Omega} \quad \text { a.e. in } \Omega
$$

(where $|\beta|^{2}=\beta_{1}^{2}+\cdots+\beta_{n}^{2}$ for $\beta \in \mathbb{R}^{n}$ and ||$_{\infty, O}$ is the usual norm on $L^{\infty}(O)$ ).

Proof. Let $x \in \Omega$ and $K \in \mathcal{T}_{h}$ such that $x \in K$. Denote by $\left(e_{1}, \ldots, e_{n}\right)$ the canonical basis of $\mathbb{R}^{n}$ and by $\hat{K}$ the convex hull of $0, e_{1}, \ldots, e_{n} ; \hat{K}$ is the reference unit $n$-simplex of $\mathbb{R}^{n}$.

By (1.3) there exists a one-to-one affine map $F_{K}$ defined on $\hat{K}$ by the following:

$\forall \hat{y} \in \hat{K}, F_{K}(\hat{y})=B_{K} \hat{y}+b_{K}$, where $B_{K}$ is an $n \times n$ invertible matrix, $b_{K} \in \mathbb{R}^{n}$, and such that $F_{K}(\hat{K})=K$.

Now, denote by $\hat{h}$ and $\hat{\rho}$, respectively, the diameter and the roundness of $\hat{K}$. By the definition of $\hat{\rho}$, one has

$$
\forall j=1, \ldots, n, \quad \exists \hat{y}_{j}, \hat{z}_{j} \in \hat{K} \quad \text { such that } \hat{\rho} e_{j}=\hat{y}_{j}-\hat{z}_{j} .
$$

Hence

$$
\left|B_{K} \hat{\rho} e_{j}\right|=\left|B_{K} \hat{y}_{j}-B_{K} \hat{z}_{j}\right|=\left|F_{K}\left(\hat{y}_{j}\right)-F_{K}\left(\hat{z}_{j}\right)\right| \leq h_{K},
$$

and thus

$$
\forall j=1, \ldots, n, \quad\left|B_{K} e_{j}\right| \leq \frac{h_{K}}{\hat{\rho}}
$$

Reversing the roles of $K$ and $\hat{K}$ we obtain

$$
\forall j=1, \ldots, n, \quad\left|B_{K}^{-1} e_{j}\right| \leq \frac{\hat{h}}{\rho_{K}}
$$

Then it is clear that $v_{h} \circ F_{K}$ is an affine and coincides with $v \circ F_{K}$ on the vertices of $\hat{K}$. Let

$$
\beta=\nabla\left(v_{h} \circ F_{K}\right)(\hat{y}) \quad \forall \hat{y} \in \hat{K} .
$$

One has $\beta \in \mathbb{R}^{n}$ and

$$
\begin{aligned}
\forall j=1, \ldots, n, \quad\left|\beta_{j}\right| & =\left|\left(v_{h} \circ F_{K}\right)\left(e_{j}\right)-\left(v_{h} \circ F_{K}\right)(0)\right| \\
& =\left|\left(v \circ F_{K}\right)\left(e_{j}\right)-\left(v \circ F_{K}\right)(0)\right| \\
& \leq\left.|| \nabla v\right|_{\infty, K}\left|F_{K}\left(e_{j}\right)-F_{K}(0)\right| \\
& =|| \nabla v \|_{\infty, K}\left|B_{K} e_{j}\right|
\end{aligned}
$$


and thus by (2.2) we obtain

$$
\forall j=1, \ldots, n, \quad\left|\beta_{j}\right| \leq \frac{h_{K}}{\hat{\rho}}\|\nabla v\|_{\infty, K}
$$

From the equality

$$
\beta=\nabla\left(v_{h} \circ F_{K}\right)(\hat{x})=\nabla v_{h}(x) \cdot B_{K}
$$

we deduce

$$
\forall j=1, \ldots, n, \quad \frac{\partial v_{h}}{\partial x_{j}}(x)=\beta \cdot B_{K}^{-1} e_{j}
$$

where "." denotes the usual scalar product in $\mathbb{R}^{n}$. Hence by (2.3)-(2.5)

$$
\forall j=1, \ldots, n, \quad\left|\frac{\partial v_{h}}{\partial x_{j}}(x)\right| \leq|\beta|\left|B_{K}^{-1} e_{j}\right| \leq\left. n^{1 / 2} \frac{h_{K}}{\hat{\rho}} \frac{\hat{h}}{\rho_{K}}|| \nabla v\right|_{\infty, K} .
$$

Squaring and adding these inequalities we obtain, by (1.2),

$$
\left|\nabla v_{h}(x)\right| \leq n \nu \frac{\hat{h}}{\hat{\rho}}\|\nabla v\|_{\infty, \Omega}
$$

which completes the proof.

Let us now recall that a function $v: \Omega \rightarrow \mathbb{R}$ is said to be piecewise affine if $v$ is continuous and if there exist disjoint open subsets $\Omega_{1}, \ldots, \Omega_{s}$ of $\Omega$ such that

$$
\begin{aligned}
& \text { (i) } \bar{\Omega}=\bigcup_{i=1}^{s} \bar{\Omega}_{i}, \\
& \text { (ii) } \nabla v \text { is constant on every } \Omega_{i}, \\
& \text { (iii) } \bar{\Omega} \backslash \bigcup_{i=1}^{s} \Omega_{i} \text { has measure } 0
\end{aligned}
$$

see [9, Chap. $2, \S 2.1$, p. 27] and [10, Chap. 10, $§ 1.2$, p. 276].

THEOREM 2.1. Assume that $\varphi$ is a Borel function, bounded on bounded subsets of $\mathbb{R}^{n}$. One has

$$
\lim _{h \rightarrow 0} \varphi_{h}^{* *}(\alpha)=\varphi^{* *}(\alpha) \quad \forall \alpha \in \mathbb{R}^{n} .
$$

Proof. Let $\epsilon>0$. We know that, if $A f f_{0}(\Omega ; \mathbb{R})$ denotes the set of piecewise affine functions from $\Omega$ into $\mathbb{R}$ vanishing on $\partial \Omega$, one has

$$
\varphi^{* *}(\alpha)=\operatorname{Inf}_{v \in A f f_{0}(\Omega ; \mathbf{R})} \frac{1}{|\Omega|} \int_{\Omega} \varphi(\alpha+\nabla v(x)) d x, \quad \alpha \in \mathbb{R}^{n}
$$

(see [9, Chap. 5, §5.1, (23), p. 206]).

Hence, there exists $v_{\epsilon} \in A f f_{0}(\Omega ; \mathbb{R})$ such that

$$
\varphi^{* *}(\alpha) \leq \frac{1}{|\Omega|} \int_{\Omega} \varphi\left(\alpha+\nabla v_{\epsilon}(x)\right) d x \leq \varphi^{* *}(\alpha)+\frac{\epsilon}{2}
$$


Since $v_{\epsilon}$ is piecewise affine, there exists disjoint open subsets $\Omega_{1}^{\epsilon}, \ldots, \Omega_{s}^{\epsilon}$ of $\Omega$ such that

$$
\bar{\Omega}=\bigcup_{i=1}^{s} \bar{\Omega}_{i}^{\epsilon}, \quad|\Omega|=\sum_{i=1}^{s}\left|\Omega_{i}^{\epsilon}\right|, \quad \text { and } v_{\epsilon} \text { is affine on every } \Omega_{i}^{\epsilon}
$$

Set

$$
C_{\epsilon}=\sup _{|\beta-\alpha| \leq c|| \nabla v_{\epsilon}||_{\infty, \Omega}}|\varphi(\beta)|
$$

(where $c$ is the constant defined in Lemma 2.1) and

$$
F_{\epsilon}=\bar{\Omega} \backslash \bigcup_{1 \leq i \leq s} \Omega_{i}^{\epsilon}
$$

$F_{\epsilon}$ is a compact set of $\mathbb{R}^{n}$ of measure zero. Then there exists an open set $O_{\epsilon}$ such that

$$
F_{\epsilon} \subset O_{\epsilon} \quad \text { and } \quad\left|O_{\epsilon}\right| \leq \frac{\epsilon|\Omega|}{4 C_{\epsilon}}
$$

Next, set

$$
h_{0}=d\left(F_{\epsilon}, \bar{\Omega} \backslash O_{\epsilon}\right),
$$

where $d$ denotes the distance function. Since $\bar{\Omega} \backslash O_{\epsilon}$ is closed and $F_{\epsilon}$ is compact, one has $h_{0}>0$. Then, consider $h$ such that $0<h<h_{0}$, and let us denote by $v_{\epsilon, h}$ the interpolate of $v_{\epsilon}$ on $\mathcal{T}_{h}$. Since $v_{\epsilon}$ is affine on every $\Omega_{i}^{\epsilon}$, the functions $v_{\epsilon, h}$ and $v_{\epsilon}$ coincide on $\Omega \backslash O_{\epsilon}$. Indeed, the $n$-simplices that intersect $F_{\epsilon}$ are contained in $O_{\epsilon}$. Therefore,

$$
\begin{aligned}
& \left|\int_{\Omega} \varphi\left(\alpha+\nabla v_{\epsilon, h}(x)\right) d x-\int_{\Omega} \varphi\left(\alpha+\nabla v_{\epsilon}(x)\right) d x\right| \\
& =\left|\int_{O_{\epsilon} \cap \Omega} \varphi\left(\alpha+\nabla v_{\epsilon, h}(x)\right) d x-\int_{O_{\epsilon} \cap \Omega} \varphi\left(\alpha+\nabla v_{\epsilon}(x)\right) d x\right| \\
& \leq\left|\int_{O_{\epsilon} \cap \Omega} \varphi\left(\alpha+\nabla v_{\epsilon, h}(x)\right) d x\right|+\left|\int_{O_{\epsilon} \cap \Omega} \varphi\left(\alpha+\nabla v_{\epsilon}(x)\right) d x\right| \\
& \left.\leq 2\left|O_{\epsilon}\right| C_{\epsilon} \quad \text { (by }(2.1) \text { and the definition of } C_{\epsilon}\right) \\
& \leq 2 \frac{\epsilon|\Omega|}{4 C_{\epsilon}} C_{\epsilon}=\frac{\epsilon|\Omega|}{2} .
\end{aligned}
$$

Combining this with (2.6) we obtain

$$
\varphi^{* *}(\alpha) \leq \frac{1}{|\Omega|} \int_{\Omega} \varphi\left(\alpha+\nabla v_{\epsilon, h}(x)\right) d x \leq \varphi^{* *}(\alpha)+\epsilon
$$

which gives

$$
\varphi^{* *}(\alpha) \leq \varphi_{h}^{* *}(\alpha) \leq \varphi^{* *}(\alpha)+\epsilon .
$$

This concludes the proof. 
Remark 2.1. The above arguments extend to the vectorial case, i.e., for

$$
\varphi: M_{m \times n} \rightarrow \mathbb{R}
$$

where $M_{m \times n}$ denotes the set of $m \times n$ matrices, if the convex envelope is replaced by the quasi-convex envelope. We refer the reader to [1] for details.

3. Geometric properties of $\varphi^{* *}$. First, let us recall some classical definitions about convex sets.

Let $C$ be a convex set of $\mathbb{R}^{N}$.

We denote by $a f f(C)$ the affine hull of $C$, that is to say, the smallest affine subspace of $\mathbb{R}^{N}$ containing $C$. The integer $\operatorname{dim} C=\operatorname{dim}(a f f(C))$ is the dimension of $C$.

A supporting hyperplane to $C$ is a hyperplane dividing the space into two closed half-spaces, one containing $C$ and a point of $C$ on its boundary. If $C$ is not $N$ dimensional, so that $\operatorname{aff}(C) \neq \mathbb{R}^{N}$, we can always extend $a f f(C)$ into a supporting hyperplane containing $C$ itself. Such a supporting hyperplane is hardly of interest, so we will only consider proper supporting hyperplanes to $C$, i.e., hyperplanes that do not contain $C$ itself.

An extreme point of $C$ is a point $x \in C$ such that there is no way to express $x$ as a convex combination $x=\lambda y+(1-\lambda) z$ with $y, z \in C$ and $0<\lambda<1$, unless we choose $y=z=x$.

An exposed point of $C$ is a point $x \in C$ through which passes a supporting hyperplane to $C$, which contains $x$ and no other point of $C$.

An exposed point is an extreme point, but the converse is false.

An extreme half-line is a half-line $D$ included in $C$, such that for all closed line segments $[y, z] \subset C$ verifying $] y, z[\cap D \neq \emptyset$, one has $[y, z] \subset D$.

We call relative interior of the convex set $C$, which we denote by $r i(C)$, the set of all points which are interior to $C$ for the topology of $a f f(C)$. of $C$.

If $\operatorname{dim} C=N$, then $r i(C)=\stackrel{\circ}{\mathrm{C}}$. The set $\bar{C} \backslash r i(C)$ is called a relative boundary

Finally, for a set $A \subset \mathbb{R}^{N}$, we denote by $\operatorname{co}(A)$ the convex hull of $A$.

Let us now recall some classical results.

Proposition 3.1. Let $C$ be a convex set of $\mathbb{R}^{N}$ and $x$ a point of the relative boundary of $C$; then there exists a proper supporting hyperplane to $\bar{C}$ containing $x$.

Proof. See [15, Part III, §11, Thm. 11.6, p. 100].

Proposition 3.2. Let $C$ be a closed convex set of $\mathbb{R}^{N}$ containing no lines, and let $S$ be the set of all extreme points and extreme half-lines of $C$. Then $C=\operatorname{co}(S)$.

Proof. See [15, Part IV, §18, Thm. 18.5, p. 100].

Proposition 3.3 (Straszewicz's theorem). Let $C$ be a closed convex set of $\mathbb{R}^{N}$. The set of exposed points of $C$ is a dense subset of the set of extreme points of $C$.

Proof. See [15, Part IV, §18, Thm. 18.6, p. 166].

Next, recall that the epigraph of a function $f: \mathbb{R}^{n} \rightarrow \mathbb{R}$ is the set defined by

$$
e p i(f)=\left\{(x, y) \in \mathbb{R}^{n} \times \mathbb{R}: y \geq f(x)\right\}
$$

We know that

$$
\operatorname{epi}\left(\varphi^{* *}\right)=\overline{c o}(\operatorname{epi}(\varphi))
$$

where $\overline{c o}(A)$ is the closed convex hull of $A$ (see [10, Chap. I, §3.2, Prop. 3.2, p. 15]). So, $\operatorname{epi}\left(\varphi^{* *}\right)$ is a closed convex set of $\mathbb{R}^{n} \times \mathbb{R}$. 
We are now going to give a simple, necessary (and clearly not sufficient) condition in order for a point $(\alpha, \beta) \in \mathbb{R}^{n} \times \mathbb{R}$ to be an extreme point of epi $\left(\varphi^{* *}\right)$.

Proposition 3.4. Assume that the function $\varphi$ is continuous. Let $(\alpha, \beta) \in \mathbb{R}^{n} \times$ R. If $(\alpha, \beta)$ is an extreme point of epi $\left(\varphi^{* *}\right)$, then

$$
\beta=\varphi^{* *}(\alpha)=\varphi(\alpha) .
$$

Proof. Since $\varphi$ is continuous, by Proposition 3.3, it is sufficient to show that (3.2) holds for the exposed points.

So, let $(\alpha, \beta)$ be an exposed point of $\operatorname{epi}\left(\varphi^{* *}\right)$. It is clear that $(\alpha, \beta)$ is a point of the (topological) boundary of the set $\operatorname{epi}\left(\varphi^{* *}\right)$, so that we have

$$
\beta=\varphi^{* *}(\alpha) \text {. }
$$

Now, assume that

$$
\varphi^{* *}(\alpha)<\varphi(\alpha)
$$

Since $\left(\alpha, \varphi^{* *}(\alpha)\right)$ is an exposed point of epi $\left(\varphi^{* *}\right)$, there exists a supporting hyperplane $H$ of $e p i\left(\varphi^{* *}\right)$ that contains $\left(\alpha, \varphi^{* *}(\alpha)\right)$ and such that

$$
H \cap \operatorname{epi}\left(\varphi^{* *}\right)=\left\{\left(\alpha, \varphi^{* *}(\alpha)\right)\right\}
$$

Thus there exists an affine function $g: \mathbb{R}^{n} \rightarrow \mathbb{R}$ such that

$$
H=\left\{(x, g(x)): x \in \mathbb{R}^{n}\right\}
$$

and

$$
g \leq \varphi^{* *}, \quad g(\alpha)=\varphi^{* *}(\alpha) .
$$

On the other hand, using the continuity of $g, \varphi$, and $\varphi^{* *}$, and (3.3), it is easy to see that there exists $\epsilon>0$ such that

$$
\forall x \in \bar{B}(\alpha, \epsilon), \quad\left\{\begin{array}{l}
\varphi^{* *}(x)-g(x) \leq \frac{\varphi(\alpha)-g(\alpha)}{2} \\
\varphi(x)-g(x) \geq \frac{\varphi(\alpha)-g(\alpha)}{2}
\end{array}\right.
$$

where $\bar{B}(\alpha, \epsilon)=\left\{x \in \mathbf{R}^{n}:|x-\alpha| \leq \epsilon\right\}$. Set

$$
\eta=\inf _{|z-\alpha|=\epsilon}\left(\varphi^{* *}(z)-g(z)\right) \text {. }
$$

Using (3.5) we see that $\eta \geq 0$.

Then, denote by $\psi$ the function $\psi=\max \left(\varphi^{* *}, g+\eta\right)$. We have

$$
\psi \text { is convex and } \psi \geq \varphi^{* *} \text {. }
$$

If $x \in \bar{B}(\alpha, \epsilon)$, then, using (3.6), we have

$$
\begin{aligned}
g(x)+\eta & \leq g(x)+\varphi^{* *}(z)-g(z) \quad(\text { for } z \text { such that }|z-\alpha|=\epsilon) \\
& \leq g(x)+\frac{\varphi(\alpha)-g(\alpha)}{2} \\
& \leq g(x)+\varphi(x)-g(x) \\
& =\varphi(x) .
\end{aligned}
$$


Since $\varphi^{* *} \leq \varphi$, we get

$$
\forall x \in \bar{B}(\alpha, \epsilon), \quad \psi(x) \leq \varphi(x)
$$

If $x \notin \bar{B}(\alpha, \epsilon)$, then choose $z$ such that $|z-\alpha|=\epsilon$ and $z \in] x, \alpha[$, i.e., $z=$ $\lambda x+(1-\lambda) \alpha, \lambda \in(0,1)$, implying that

$$
\varphi^{* *}(z) \leq \lambda \varphi^{* *}(x)+(1-\lambda) \varphi^{* *}(\alpha) .
$$

Using (3.5) we obtain

$$
\begin{aligned}
\varphi^{* *}(x) & \geq \frac{1}{\lambda}\left(\varphi^{* *}(z)-(1-\lambda) g(\alpha)\right) \\
& =\frac{1}{\lambda}\left(\varphi^{* *}(z)-g(z)+\lambda g(x)\right) \\
& \geq g(x)+\frac{1}{\lambda} \eta \\
& \geq g(x)+\eta,
\end{aligned}
$$

and thus

$$
\forall x \notin \bar{B}(\alpha, \epsilon), \quad \psi(x)=\varphi^{* *}(x) \leq \varphi(x) .
$$

Then (3.8) and (3.9) show that $\psi \leq \varphi$, and by (3.7) and the fact that $\varphi^{* *}$ is the greatest convex functions lying below $\varphi$, we get $\psi=\varphi^{* *}$, which also reads

$$
\forall x \in \mathbb{R}^{n}, \quad g(x)+\eta \leq \varphi^{* *}(x) .
$$

In particular,

$$
\varphi^{* *}(\alpha)=g(\alpha) \leq \varphi^{* *}(\alpha)-\eta \quad \text { with } \eta \geq 0 .
$$

Thus, $\eta=0$. Therefore, since $\left\{z \in \mathbb{R}^{n}:|z-\alpha|=\epsilon\right\}$ is compact and since $\varphi^{* *}-g$ is continuous, there exists $z_{0}$ such that

$$
\left|z_{0}-\alpha\right|=\epsilon \text { and } \varphi^{* *}\left(z_{0}\right)=g\left(z_{0}\right) .
$$

Then both points $\left(\alpha, \varphi^{* *}(\alpha)\right) \neq\left(z_{0}, \varphi^{* *}\left(z_{0}\right)\right)$ belong to $H \cap \operatorname{epi}\left(\varphi^{* *}\right)$, which contradicts (3.4).

The next lemma will be used in the proof of Proposition 3.5 below.

LEMMA 3.1. Let $\psi: \mathbb{R}^{n} \rightarrow \mathbb{R}$ be a convex function, $a_{1}, \ldots, a_{k} \in \mathbb{R}^{n}$, and suppose there exist $\lambda_{1}, \ldots, \lambda_{k}>0$ such that $\lambda_{1}+\cdots+\lambda_{k}=1$ and

$$
\psi\left(\sum_{i=1}^{k} \lambda_{i} a_{i}\right)=\sum_{i=1}^{k} \lambda_{i} \psi\left(a_{i}\right)
$$

Then, $\psi$ is affine on $c o\left\{a_{1}, \ldots, a_{k}\right\}$.

Proof. Set $a=\sum_{i=1}^{k} \lambda_{i} a_{i}$. We know that one can find an affine function $g$ such that

$$
g \leq \psi, \quad g(a)=\psi(a) .
$$


Then

$$
g\left(a_{i}\right)=\psi\left(a_{i}\right) \quad \forall i=1, \ldots, k .
$$

Indeed, if this is not the case we would have

$$
g(a)=\sum_{i=1}^{k} \lambda_{i} g\left(a_{i}\right)<\sum_{i=1}^{k} \lambda_{i} \psi\left(a_{i}\right)=\psi(a)
$$

which is impossible. But then for any

$$
y=\sum_{i=1}^{k} \mu_{i} a_{i}, \quad \mu_{i} \geq 0, \quad \sum_{i=1}^{k} \mu_{i}=1
$$

one has

$$
\psi(y) \leq \sum_{i=1}^{k} \mu_{i} \psi\left(a_{i}\right)=\sum_{i=1}^{k} \mu_{i} g\left(a_{i}\right)=g(y)
$$

and thus by (3.11) $\psi$ and $g$ agree on $c o\left\{a_{1}, \ldots, a_{k}\right\}$.

Now, denote by $\mathcal{A}_{\varphi}$ the set defined by

$$
\mathcal{A}_{\varphi}=\left\{a \in \mathbb{R}^{n}: \varphi(a)=\varphi^{* *}(a)\right\} .
$$

We now have the following.

Proposition 3.5. Suppose that $\varphi$ is continuous and that epi $\left(\varphi^{* *}\right)$ does not contain any line or any extreme half-line. Let $\alpha \in \mathbb{R}^{n}$. Then, either $\alpha \in \mathcal{A}_{\varphi}$ or there exists $a_{1}, \ldots, a_{k} \in \mathcal{A}_{\varphi}$ such that

$$
\alpha \in \operatorname{ri}\left(\operatorname{co}\left\{a_{1}, \ldots, a_{k}\right\}\right) \text { and } \varphi^{* *} \text { is affine on } \operatorname{co}\left\{a_{1}, \ldots, a_{k}\right\} .
$$

Proof. Assume $\alpha \notin \mathcal{A}_{\varphi}$. Since epi $\left(\varphi^{* *}\right)$ does not contain any line or extreme halfline and is a closed convex set, one can apply Proposition 3.2 and claim that $\left(\alpha, \varphi^{* *}(\alpha)\right)$ is in the convex hull of the extreme points of epi $\left(\varphi^{* *}\right)$. Hence, by Proposition 3.4, there exist $a_{1}, \ldots, a_{k} \in \mathcal{A}_{\varphi}$ such that

$$
\left(\alpha, \varphi^{* *}(\alpha)\right)=\sum_{i=1}^{k} \lambda_{i}\left(a_{i}, \varphi\left(a_{i}\right)\right)
$$

with

$$
\lambda_{i}>0, \quad \sum_{i=1}^{k} \lambda_{i}=1 \quad\left(\text { and } k \geq 2 \text { since } \alpha \notin \mathcal{A}_{\varphi}\right)
$$

Hence

$$
\alpha=\sum_{i=1}^{k} \lambda_{i} a_{i} \text { and } \alpha \in \operatorname{ri}\left(\operatorname{co}\left\{a_{1}, \ldots, a_{k}\right\}\right)
$$

Moreover,

$$
\varphi^{* *}(\alpha)=\sum_{i=1}^{k} \lambda_{i} \varphi^{* *}\left(a_{i}\right)
$$

Then, applying Lemma 3.1 , we obtain that $\varphi^{* *}$ is affine on $c o\left\{a_{1}, \ldots, a_{k}\right\}$. 
In order to be in a position to apply Proposition 3.5, we can impose some growth condition on $\varphi$. Indeed, we have the following.

Proposition 3.6. If $\varphi$ satisfies

$$
\lim _{|\beta| \rightarrow+\infty} \frac{\varphi(\beta)}{|\beta|}=+\infty
$$

then epi $\left(\varphi^{* *}\right)$ does not contain any half-line on its boundary.

Proof. The property (3.13) implies that $\varphi$ is bounded from below. So, without loss of generality, we can assume $\varphi \geq 0$. Next, suppose that the boundary of $\operatorname{epi}\left(\varphi^{* *}\right)$ contains a half-line. Then, there exists a half-line $D \subset \mathbb{R}^{n}$ such that $\varphi^{* *}$ restricted to $D$ is affine.

Let $a \in \mathcal{A}_{\varphi}$ be the origin of $D$. Replacing $\varphi$ by

$$
\tilde{\varphi}: \beta \rightarrow \varphi(Q \cdot \beta+a)
$$

where $Q$ is some orthogonal matrix, one can suppose that $D=[0,+\infty) \times\{0\} \times \cdots \times\{0\}$, and that $a=0 \in \mathcal{A}_{\varphi}$.

Then there exists $\lambda \geq 0$ such that

$$
\forall \beta=\left(\beta_{1}, 0, \ldots, 0\right) \in D, \quad \text { one has } \varphi^{* *}(\beta)=\lambda \beta_{1}+\varphi(0) .
$$

By (3.13) there exists $b>0$ such that

$$
\forall|\beta| \geq b, \quad \varphi(\beta) \geq(\lambda+1)|\beta| .
$$

Thus, in particular, if $\beta=\left(\beta_{1}, \ldots, \beta_{n}\right)$ satisfies $\beta_{1} \geq b$, we will have $|\beta| \geq b$, and so

$$
\varphi(\beta) \geq(\lambda+1)|\beta| \geq(\lambda+1) \beta_{1} .
$$

Therefore,

$$
\forall \beta_{1} \geq b \quad(\lambda+1)\left(\beta_{1}-b\right) \leq(\lambda+1) \beta_{1} \leq \varphi(\beta)
$$

and

$$
\forall \beta_{1} \leq b \quad(\lambda+1)\left(\beta_{1}-b\right) \leq 0 \leq \varphi(\beta) .
$$

So we have

$$
\forall \beta \in \mathbb{R}^{n} \quad(\lambda+1)\left(\beta_{1}-b\right) \leq \varphi(\beta) .
$$

Since the function on the left-hand side of the above inequality is affine, we deduce

$$
\forall \beta \in \mathbb{R}^{n} \quad(\lambda+1)\left(\beta_{1}-b\right) \leq \varphi^{* *}(\beta) .
$$

In particular,

$$
\forall \beta \in D \quad(\lambda+1)\left(\beta_{1}-b\right) \leq \lambda \beta_{1}+\varphi(0)
$$

i.e.,

$$
\forall \beta_{1} \geq 0, \quad \beta_{1} \leq \varphi(0)+(\lambda+1) b .
$$

This is impossible since the right-hand side is constant. Consequently, epi( $\left.\varphi^{* *}\right)$ cannot contain a half-line on its boundary. 
Remark 3.1. The assumption (3.13) is just a sufficient condition in order for the boundary of $\operatorname{epi}\left(\varphi^{* *}\right)$ to not contain a half-line. Indeed, consider a function $\varphi$ whose graph is a branch of a hyperbola. For instance,

$$
\varphi(\beta)=\sqrt{1+\beta^{2}}
$$

Then, in this case,

$$
\varphi^{* *}=\varphi, \quad \lim _{|\beta| \rightarrow+\infty} \frac{\varphi(\beta)}{|\beta|}=1
$$

and the graph of $\varphi^{* *}$ does not contain any half-line.

4. Estimate of $\varphi_{h}^{* *}-\varphi^{* *}$.

THEOREM 4.1. Let $\psi: \mathbb{R}^{n} \rightarrow \mathbb{R}$ be a nonnegative function bounded on bounded subsets of $\mathbb{R}^{n}$. Let $\alpha \in \mathbb{R}^{n}$, such that $\alpha \in \operatorname{co}\left(\psi^{-1}(\{0\})\right)$. Then

$$
\psi^{* *}(\alpha)=0 \text { and } \psi_{h}^{* *}(\alpha) \leq C h^{1 / 2},
$$

where $C$ is a constant depending on $\Omega, \psi$, and $\alpha$.

Proof. See [3, §2].

Remark 4.1. We do not know if the estimate (4.1) is sharp. One can find in [4] the idea of the proof of Theorem 4.1 in the case where $\alpha=\lambda \omega_{1}+(1-\lambda) \omega_{2}$, with $0<\lambda<1$ and $\psi\left(\omega_{1}\right)=\psi\left(\omega_{2}\right)=0$ ("two well problem").

Let us recall that $\mathcal{A}_{\varphi}=\left\{a \in \mathbb{R}^{n}: \varphi(a)=\varphi^{* *}(a)\right\}$.

THEOREM 4.2. Assume that $\varphi$ is bounded on bounded subsets of $\mathbb{R}^{n}$, and let $\alpha \in \mathbb{R}^{n}$. Suppose there exists $a_{1}, \ldots, a_{k} \in \mathcal{A}_{\varphi}$ such that

$$
\alpha \in \operatorname{ri}\left(\operatorname{co}\left\{a_{1}, \ldots, a_{k}\right\}\right) \text { and } \varphi^{* *} \text { is affine on } \operatorname{co}\left\{a_{1}, \ldots, a_{k}\right\} \text {. }
$$

Then

$$
0 \leq \varphi_{h}^{* *}(\alpha)-\varphi^{* *}(\alpha) \leq C h^{1 / 2}
$$

where $C$ is a constant depending on $\Omega, \varphi$, and $\alpha$.

Proof. Let $H$ be a supporting hyperplane to epi $\left(\varphi^{* *}\right)$ containing $\left(\alpha, \varphi^{* *}(\alpha)\right)$; see Proposition 3.1.

Let $g: \mathbb{R}^{n} \rightarrow \mathbb{R}$ be the affine function such that $H=\left\{(x, g(x)): x \in \mathbb{R}^{n}\right\}$, and

$$
g(\alpha)=\varphi^{* *}(\alpha) \text { and } g \leq \varphi^{* *} .
$$

From (4.2) we deduce that

$$
\alpha=\sum_{i=1}^{k} \lambda_{i} a_{i} \quad \text { with } \lambda_{i}>0
$$

and

$$
\varphi^{* *}(\alpha)=\sum_{i=1}^{k} \lambda_{i} \varphi^{* *}\left(a_{i}\right)
$$

Now, suppose there exists $l \in\{1, \ldots, k\}$ such that $g\left(a_{l}\right)<\varphi^{* *}\left(a_{l}\right)$. Then, since $\lambda_{l}>0$, one has

$$
g(\alpha)=\sum_{i=1}^{k} \lambda_{i} g\left(a_{i}\right)<\sum_{i=1}^{k} \lambda_{i} \varphi^{* *}\left(a_{i}\right)=\varphi^{* *}(\alpha)=g(\alpha)
$$


hence a contradiction. Consequently, (4.3) implies that $g$ and $\varphi^{* *}$ coincide at $\alpha, a_{1}, \ldots$, $a_{k}$, and thus on the whole set $c o\left\{a_{1}, \ldots, a_{k}\right\}$.

Next, set $\psi=\varphi-g$. Clearly, one has

$$
\psi \geq 0 \text { and } \quad \psi\left(a_{i}\right)=0 \quad \forall i=1, \ldots, k .
$$

Then, using Theorem 4.1, we obtain

$$
\psi_{h}^{* *}(\alpha) \leq C h^{1 / 2}
$$

But thanks to Remark 1.1 we can write

$$
\psi_{h}^{* *}(\alpha)=(\varphi-g)_{h}^{* *}(\alpha)=\varphi_{h}^{* *}(\alpha)-g(\alpha)
$$

and thus, since $g(\alpha)=\varphi^{* *}(\alpha),(4.4)$ becomes

$$
\varphi_{h}^{* *}(\alpha)-\varphi^{* *}(\alpha) \leq C h^{1 / 2}
$$

and the proof is complete.

The condition (4.2) is a technical assumption. Thanks to Propositions 3.5 and 3.6 it can be relaxed. Indeed, we have the following.

Corollary 4.1. Suppose $\varphi$ is continuous and epi $\left(\varphi^{* *}\right)$ does not contain any line or extreme half-line. Then for all $\alpha \in \mathbb{R}^{n}$ one has

$$
0 \leq \varphi_{h}^{* *}(\alpha)-\varphi^{* *}(\alpha) \leq C h^{1 / 2}
$$

where $C$ is a constant depending on $\Omega, \varphi$, and $\alpha$.

This is, in particular, the case when

$$
\lim _{|\beta| \rightarrow+\infty} \frac{\varphi(\beta)}{|\beta|}=+\infty
$$

Proof. This follows from Theorem 4.2 and Propositions 3.5 and 3.6.

Remark 4.2. The interesting feature of the second part of the corollary lies in the fact that our hypotheses concern $\varphi$ only (compare to Theorem 4.2).

5. The one-dimensional case. In this part, we are going to specify $n=1$. Our results are then more precise. They will also focus on the difficulties when trying to extend Corollary 4.1. First, let us prove two lemmas.

LEMMA 5.1. Let $\varphi: \mathbb{R} \rightarrow \mathbb{R}$ be a continuous function. Let $a \in \mathbb{R}$ be such that $\varphi^{* *}(a)=\varphi(a)$. If there exists $b \in[-\infty,+\infty]$ such that

$$
\varphi^{* *}(x)<\varphi(x) \quad \forall x \in(a, b) \quad(\text { or }(b, a))
$$

then $\varphi^{* *}$ is affine on $(a, b)$.

Proof. This is well known. See also [2], [12], and [13].

LEMMA 5.2. Let $\psi: \mathbb{R} \rightarrow \mathbb{R}$ be a nonnegative function, bounded on bounded subsets of $\mathbb{R}$. Let $\omega_{1}, \omega_{2} \in \mathbb{R}$ be such that

$$
\psi\left(\omega_{1}\right)=\psi\left(\omega_{2}\right)=0
$$


Let $\alpha \in\left[\omega_{1}, \omega_{2}\right] ;$ then

$$
\psi^{* *}(\alpha)=0 \text { and } \psi_{h}^{* *}(\alpha) \leq \frac{C}{|\Omega|} h,
$$

where $C$ is a constant depending on $\psi$ and $\alpha$.

Proof. Suppose $\Omega=\left(y_{1}, y_{2}\right)$ and define

$$
u=\min \left(\left(\omega_{2}-\alpha\right)\left(x-y_{1}\right),\left(\omega_{1}-\alpha\right)\left(x-y_{2}\right)\right) .
$$

The triangulation $\mathcal{T}_{h}$ of $\Omega$ is now a subdivision

$$
x_{0}<x_{1}<\cdots<x_{p} \quad \text { with } x_{0}=y_{1}, x_{p}=y_{2}, \quad \text { and } \quad\left|x_{i}-x_{i-1}\right| \leq h .
$$

Let $u_{h}$ be the interpolate of $u$ on $\mathcal{T}_{h}$. Clearly, $u_{h}$ and $u$ coincide, except maybe on some interval $\left(x_{k}, x_{k+1}\right)$, and if $u_{h}^{\prime}(x)=\beta$ on $\left(x_{k}, x_{k+1}\right)$ we have

$$
|\Omega| \psi_{h}^{* *}(\alpha) \leq \int_{\Omega} \psi\left(\alpha+u_{h}^{\prime}(x)\right) d x=\int_{x_{k}}^{x_{k+1}} \psi\left(\alpha+u_{h}^{\prime}(x)\right) d x \leq \psi(\alpha+\beta) h .
$$

This concludes the proof of the lemma, since

$\beta=\frac{1}{x_{k+1}-x_{k}} \int_{x_{k}}^{x_{k+1}} u_{h}^{\prime}(x) d x=\frac{1}{x_{k+1}-x_{k}} \int_{x_{k}}^{x_{k+1}} u^{\prime}(x) d x \in\left(\omega_{1}-\alpha, \omega_{2}-\alpha\right)$.

Remark 5.1. Lemma 5.2 improves, in the one-dimensional case, the estimate (4.1). Moreover, (5.1) is sharp. Indeed, assume

$$
\Omega=(0,1) \quad \text { and } \quad \omega_{1}=-1, \quad \omega_{2}=1 .
$$

Let $h>0$ and $l \in \mathbf{N}^{*}$ be such that $h=1 /(2 l+1)$. Consider the subdivision of $\Omega$ defined by

$$
x_{k}=\frac{k}{2 l+1}, \quad 0 \leq k \leq 2 l+1
$$

If $u_{h} \in V_{h}^{0}$, one has

$$
\int_{\Omega} \varphi\left(u_{h}^{\prime}(x)\right) d x=\sum_{k=1}^{2 l+1} \frac{1}{2 l+1} \varphi\left(a_{k}\right)=h \sum_{k=1}^{2 l+1} \varphi\left(a_{k}\right),
$$

where

$$
a_{k}=u_{h}^{\prime}(x) \quad \forall x \in\left(x_{k-1}, x_{k}\right), \quad k=1, \ldots, 2 l+1
$$

Moreover,

$$
\sum_{k=1}^{2 l+1} a_{k}=\int_{0}^{1} u_{h}^{\prime}(x) d x=0 .
$$

Suppose that $\{1, \ldots, 2 l+1\}$ can be split into two subsets, $I_{1}$ and $I_{2}$, such that

$$
\begin{aligned}
& \forall k \in I_{1}, \quad a_{k} \in\left(-1-\frac{1}{2 l+1},-1+\frac{1}{2 l+1}\right), \\
& \forall k \in I_{2}, \quad a_{k} \in\left(1-\frac{1}{2 l+1}, 1+\frac{1}{2 l+1}\right) .
\end{aligned}
$$


Set $n_{i}=\operatorname{card} I_{i}, i=1,2$. One clearly has $n_{1}+n_{2}=2 l+1$ and $\left|n_{1}-n_{2}\right| \geq 1$.

If $n_{2}-n_{1} \geq 1$

$$
\begin{aligned}
\sum_{k=1}^{2 l+1} a_{k} & =\sum_{k \in I_{1}} a_{k}+\sum_{k \in I_{2}} a_{k} \\
& >n_{1}\left(-1-\frac{1}{2 l+1}\right)+n_{2}\left(1-\frac{1}{2 l+1}\right) \\
& =n_{2}-n_{1}-1 \geq 0
\end{aligned}
$$

which implies that $\sum_{k=1}^{2 l+1} a_{k}>0$ and contradicts (5.3). If $n_{1}-n_{2} \geq 1$, a similar argument would lead to the same contradiction. Thus, the splitting (5.4) is not possible and there must exist some $k_{0} \in\{1, \ldots, 2 l+1\}$ for which

$$
\left|a_{k_{0}}-1\right| \geq \frac{1}{2 l+1} \text { and }\left|a_{k_{0}}+1\right| \geq \frac{1}{2 l+1}
$$

Let us then consider, for $s>0$,

$$
\varphi_{s}: \mathbb{R} \rightarrow \mathbb{R}_{+} \text {defined by } \varphi_{s}(\beta)=\min \left(|\beta-1|^{s},|\beta+1|^{s}\right) .
$$

One has $\varphi_{s}\left(a_{k_{0}}\right) \geq h^{s}$ and, by (5.2),

$$
\int_{\Omega} \varphi_{s}\left(u_{h}^{\prime}(x)\right) d x \geq h^{1+s} \quad \forall u_{h} \in V_{h}^{0}
$$

This shows that the estimate (5.1) cannot be improved, in general, by a stronger power of $h$ on the right-hand side.

As above, set

$$
\mathcal{A}_{\varphi}=\left\{a \in \mathbb{R}: \varphi(a)=\varphi^{* *}(a)\right\} .
$$

The following theorem is the main result of this section.

TheOREM 5.1. Suppose that $\varphi$ is continuous. If $\alpha \in \operatorname{co}\left(\mathcal{A}_{\varphi}\right)$, then

$$
0 \leq \varphi_{h}^{* *}(\alpha)-\varphi^{* *}(\alpha) \leq \frac{C}{|\Omega|} h
$$

where $C$ is a constant depending on $\varphi$ and $\alpha$.

Proof. If $\alpha \in \mathcal{A}_{\varphi}$, the result is clear by taking $v=0$ in (1.4). So, assume $\alpha \in \operatorname{co}\left(\mathcal{A}_{\varphi}\right) \backslash \mathcal{A}_{\varphi}$, and more precisely $\alpha \in\left(a_{1}, a_{2}\right)$, where $a_{1}, a_{2} \in \mathcal{A}_{\varphi}$ satisfy

$$
\forall a \in\left(a_{1}, a_{2}\right), \quad \varphi^{* *}(a)<\varphi(a) .
$$

By Lemma 5.1, $\varphi^{* *}$ is affine on $\left[a_{1}, a_{2}\right]$. Let us denote by $g$ the affine function, which agrees with $\varphi^{* *}$ on $\left[a_{1}, a_{2}\right]$. One has $\varphi^{* *} \geq g$ and, if we set $\psi=\varphi-g$, we have $\psi \geq 0$ together with $\psi\left(a_{1}\right)=\psi\left(a_{2}\right)=0$. We can then apply Lemma 5.2 to get

$$
\psi_{h}^{* *}(\alpha) \leq \frac{C}{|\Omega|} h
$$


Now, taking Remark 1.1 into account, we deduce

$$
\psi_{h}^{* *}(\alpha)=(\varphi-g)_{h}^{* *}(\alpha)=\varphi_{h}^{* *}(\alpha)-g(\alpha),
$$

which gives, by (5.5) and since $g(\alpha)=\varphi^{* *}(\alpha)$,

$$
\varphi_{h}^{* *}(\alpha)-\varphi^{* *}(\alpha) \leq \frac{C}{|\Omega|} h .
$$

This concludes the proof.

COROLlaRY 5.1. Suppose that $\varphi$ is continuous and verifies

$$
\lim _{|\beta| \rightarrow+\infty} \frac{\varphi(\beta)}{|\beta|}=+\infty
$$

Then,

$$
\forall \alpha \in \mathbb{R}, \quad 0 \leq \varphi_{h}^{* *}(\alpha)-\varphi^{* *}(\alpha) \leq \frac{C}{|\Omega|} h,
$$

where $C$ is a constant depending on $\varphi$ and $\alpha$.

Proof. From (5.6) we see that $\varphi$ is bounded from below. We can then assume $\varphi \geq 0$. Next, by Theorem 5.1 , it is enough to prove that $\operatorname{co}\left(\mathcal{A}_{\varphi}\right)=\mathbb{R}$. So, suppose $\operatorname{co}\left(\mathcal{A}_{\varphi}\right) \neq \mathbb{R}$. By replacing, if necessary, $\varphi$ by $\check{\varphi}: \beta \rightarrow \varphi(-\beta)$, one can assume

$$
a_{1}=\sup \mathcal{A}_{\varphi}<+\infty \text { and } \operatorname{co}\left(\mathcal{A}_{\varphi}\right) \subset\left(-\infty, a_{1}\right]
$$

Therefore,

$$
\forall \beta>a_{1}, \varphi^{* *}(\beta)<\varphi(\beta) \text { and since } \mathcal{A}_{\varphi} \text { is closed, } \varphi^{* *}\left(a_{1}\right)=\varphi\left(a_{1}\right)
$$

Applying Lemma 5.1, we deduce that $\varphi^{* *}$ is affine on $\left[a_{1},+\infty\right)$, that is to say

$$
\forall \beta \geq a_{1}, \quad \varphi^{* *}(\beta)=\lambda\left(\beta-a_{1}\right)+\varphi\left(a_{1}\right) \text { for some } \lambda \geq 0 .
$$

Now (5.6) implies that there exists $a_{2} \geq 0$ such that

$$
\forall \beta \geq a_{2}, \quad \varphi(\beta) \geq(\lambda+1) \beta .
$$

Then

$$
\begin{array}{ll}
\forall \beta \leq a_{2} & (\lambda+1)\left(\beta-a_{2}\right) \leq 0 \leq \varphi(\beta) \quad \text { and } \\
\forall \beta \geq a_{2} & (\lambda+1)\left(\beta-a_{2}\right) \leq(\lambda+1) \beta \leq \varphi(\beta) .
\end{array}
$$

So,

$$
\forall \beta \in \mathbb{R}, \quad(\lambda+1)\left(\beta-a_{2}\right) \leq \varphi(\beta) \Rightarrow \forall \beta \in \mathbb{R}, \quad(\lambda+1)\left(\beta-a_{2}\right) \leq \varphi^{* *}(\beta) .
$$

In particular, we have

$$
\begin{gathered}
\forall \beta \geq a_{1} \quad(\lambda+1)\left(\beta-a_{2}\right) \leq \lambda\left(\beta-a_{1}\right)+\varphi\left(a_{1}\right), \quad \text { i.e. } \\
\beta \leq(\lambda+1) a_{2}-\lambda a_{1}+\varphi\left(a_{1}\right) .
\end{gathered}
$$


This is impossible, since the right-hand side of the above inequality is constant. Therefore, $\operatorname{co}\left(\mathcal{A}_{\varphi}\right)=\mathbb{R}$, and the proof is complete.

We would like to investigate now what can be said about

$$
\varphi_{h}^{* *}(\alpha)-\varphi^{* *}(\alpha)
$$

when $\operatorname{co}\left(\mathcal{A}_{\varphi}\right) \neq \mathbb{R}$ and $\alpha \notin \operatorname{co}\left(\mathcal{A}_{\varphi}\right)$. So, suppose $\operatorname{co}\left(\mathcal{A}_{\varphi}\right) \neq \mathbb{R}$. As we have seen in the proof of Corollary 5.1, we can assume that $\operatorname{co}\left(\mathcal{A}_{\varphi}\right) \subset\left(-\infty, a_{1}\right]$ with $a_{1} \in \mathcal{A}_{\varphi}$, and that there exists $\lambda \in \mathbb{R}$ such that

$$
\forall \beta \geq a_{1}, \quad \varphi^{* *}(\beta)=\lambda\left(\beta-a_{1}\right)+\varphi\left(a_{1}\right) .
$$

In the case where (5.6) fails, we must have

$$
\liminf _{\beta \rightarrow+\infty} \frac{\varphi(\beta)}{\beta}<+\infty \text {. }
$$

More precisely, we have the following.

Proposition 5.1. Suppose that $\varphi$ is continuous and $\operatorname{co}\left(\mathcal{A}_{\varphi}\right) \subset\left(-\infty, a_{1}\right]$ with $a_{1} \in \mathcal{A}_{\varphi}$. Then

$$
\liminf _{\beta \rightarrow+\infty} \frac{\varphi(\beta)-\varphi^{* *}(\beta)}{\beta}=\inf _{\beta \geq A} \frac{\varphi(\beta)-\varphi^{* *}(\beta)}{\beta}=0 \quad \forall A>0 .
$$

Proof. Recalling that $\varphi \geq \varphi^{* *}$, if (5.8) fails, then for some $A>0$ large enough and $\epsilon>0$ one has

$$
\forall \beta \geq A \quad \varphi(\beta)-\varphi^{* *}(\beta) \geq \epsilon \beta \geq \epsilon(\beta-A) ;
$$

and since $\varphi(\beta)-\varphi^{* *}(\beta) \geq \epsilon(\beta-A)$ is obvious for $\beta<A$, we see that

$$
\forall \beta \in \mathbb{R}, \quad \varphi(\beta)-\varphi^{* *}(\beta) \geq \epsilon(\beta-A)
$$

or

$$
\forall \beta \in \mathbb{R}, \quad \varphi(\beta) \geq \varphi^{* *}(\beta)+\epsilon(\beta-A) .
$$

Since the function in the right-hand side of the above inequality is convex, one has

$$
\forall \beta \in \mathbb{R}, \quad \varphi^{* *}(\beta) \geq \varphi^{* *}(\beta)+\epsilon(\beta-A),
$$

which gives

$$
\forall \beta \in \mathbb{R}, \quad \epsilon(\beta-A) \leq 0
$$

and a contradiction.

In the case where we can control $\varphi-\varphi^{* *}$, it is still possible to get an estimate of $\varphi_{h}^{* *}-\varphi^{* *}$ in terms of a power of $h$. We will consider the case where there exists $C_{1}, C_{2}>0$, and $p \in[0,1)$ such that

$$
\forall \beta \geq a_{1}, \quad \varphi(\beta)-\varphi^{* *}(\beta) \leq C_{1}|\beta|^{p}+C_{2} .
$$

Remark 5.2. Of course, it is not always possible to get an estimate like in (5.9) as we see immediately by considering $\varphi$ such that

$$
\varphi(\beta)=\frac{\beta}{\ln (\beta)}, \quad \beta>1 .
$$


Having some control over $\varphi-\varphi^{* *}$, we can prove the following.

THEOREM 5.2. Suppose that $\varphi$ is continuous and such that $\operatorname{co}\left(\mathcal{A}_{\varphi}\right) \subset\left(-\infty, a_{1}\right]$ with $a_{1} \in \mathcal{A}_{\varphi}$, and that (5.9) holds. Let $\alpha>a_{1}$. Then

$$
0 \leq \varphi_{h}^{* *}(\alpha)-\varphi^{* *}(\alpha) \leq C h^{1-p}
$$

for some constant $C$ depending on $\Omega, C_{1}, C_{2}, p$, and $\alpha$.

Proof. Replacing, if necessary, $\varphi$ by

$$
\tilde{\varphi}: \beta \rightarrow \varphi(\alpha+\beta),
$$

one can suppose that $\alpha=0$, and thus $a_{1}<0$.

Set $\Omega=\left(y_{1}, y_{2}\right)$ and $\psi=\varphi-g$, where $g$ is the affine function that coincides with $\varphi^{* *}$ on $\left[a_{1},+\infty\right)$; see (5.7). One has $\psi \geq 0$ and $\psi\left(a_{1}\right)=0$.

Let $u$ be defined on $\Omega$ by

$$
\left\{\begin{array}{l}
u(x)=a_{1}\left(x-y_{1}\right) \quad \forall x \in\left(y_{1}, y_{2}-h\right], \\
u\left(y_{2}\right)=0 \\
u \quad \text { is affine on }\left[y_{2}-h, y_{2}\right) .
\end{array}\right.
$$

Then, denote by $u_{h}$ the interpolate of $u$ on $\mathcal{T}_{h} . u_{h} \in V_{h}^{0}$ and

$$
\begin{cases}\forall x \in\left(y_{1}, y_{2}-2 h\right), & u_{h}^{\prime}(x)=a_{1}, \\ \forall x \in\left(y_{2}-2 h, y_{2}\right), & a_{1} \leq u_{h}^{\prime}(x) \leq \frac{-a_{1}|\Omega|}{h} .\end{cases}
$$

Hence, by (5.9), we obtain

$$
\begin{aligned}
\int_{\Omega} \psi\left(u_{h}^{\prime}(x)\right) d x & =\int_{y_{2}-2 h}^{y_{2}} \psi\left(u_{h}^{\prime}(x)\right) d x \\
& \leq 2 h\left(C_{1}\left(\frac{-a_{1}|\Omega|}{h}\right)^{p}+C_{2}\right) \\
& \leq C_{3} h^{1-p} .
\end{aligned}
$$

Hence, taking Remark 1.1 into account,

$$
\varphi_{h}^{* *}(0)-g(0)=\psi_{h}^{* *}(0) \leq C h^{1-p} .
$$

The proof is complete, since $g(0)=\varphi^{* *}(0)$.

Remark 5.3. If (5.6) or (5.9) fail, we would like to show now that, in general, we cannot hope for an estimate of $\varphi_{h}^{* *}(\alpha)-\varphi^{* *}(\alpha)$ when $\alpha \notin \operatorname{co}\left(\mathcal{A}_{\varphi}\right)$ in terms of $h^{s}$. Indeed, consider the function $\varphi: \mathbb{R} \rightarrow \mathbb{R}$ defined by (see Remark 5.2)

$$
\varphi(\beta)= \begin{cases}\frac{1}{8}(\beta+1)^{2} & \text { if } \beta \leq 0 \\ \frac{\left(\beta+e^{2}\right)}{\ln \left(\beta+e^{2}\right)}+\frac{1-4 e^{2}}{8} & \text { if } \beta \geq 0 .\end{cases}
$$


It is clear that

$$
\varphi^{* *}(\beta)= \begin{cases}\frac{1}{8}(\beta+1)^{2} & \text { if } \beta \leq-1, \\ 0 & \text { if } \beta \geq-1 .\end{cases}
$$

$\varphi$ does not satisfy (5.6) and (5.9). Moreover $\operatorname{co}\left(\mathcal{A}_{\varphi}\right)=\mathcal{A}_{\varphi}=(-\infty,-1]$. It is also easy to see that $\varphi$ is $C^{1}$ on $\mathbb{R}$, and that

$$
\varphi^{\prime}(\beta)= \begin{cases}\frac{1}{4}(\beta+1) & \text { if } \beta \leq 0, \\ \frac{\ln \left(\beta+e^{2}\right)-1}{\left(\ln \left(\beta+e^{2}\right)\right)^{2}} & \text { if } \beta \geq 0 .\end{cases}
$$

Let $\Omega=(0,1), l \in \mathbf{N}^{*}$, and $h>0$ be such that $h=1 / l$. Denote by $\mathcal{T}_{h}$ the subdivision of $\Omega$ defined by

$$
x_{k}=\frac{k}{l}, \quad 0 \leq k \leq l
$$

If $u_{h} \in V_{h}^{0}$, then one has

$$
\int_{\Omega} \varphi\left(u_{h}^{\prime}(x)\right) d x=\frac{1}{l} \sum_{k=1}^{l} \varphi\left(b_{k}\right)
$$

where

$$
\forall x \in\left(x_{k-1}, x_{k}\right), \quad u_{h}^{\prime}(x)=b_{k}, \quad k=1, \ldots, l .
$$

It then follows that

$$
\varphi_{h}^{* *}(0)=h . \inf _{b \in P} \sum_{k=1}^{l} \varphi\left(b_{k}\right)
$$

where $P=\left\{b \in \mathbb{R}^{l} ; b_{1}+\cdots+b_{l}=0\right\}$. Since $\varphi(\beta) \rightarrow+\infty$ when $|\beta| \rightarrow+\infty$, the infimum in (5.10) is achieved for some point $a \in P$. Because function $\varphi$ is $C^{1}$ on $\mathbb{R}$, there exists a Lagrange multiplier $\lambda=\lambda(l)$ such that

$$
\varphi^{\prime}\left(a_{k}\right)=\lambda \quad \forall k=1, \ldots, l .
$$

It is clear that $a \neq 0$. Thus the fact that $a \in P$ implies that some of the $a_{k}$ 's are positive and some negative. Consequently, (5.11) shows that $\varphi^{\prime-1}(\{\lambda\})$ must contain at least two points. But, we see that $\varphi^{\prime}$ is increasing from $-\infty$ to $\frac{1}{4}$ on $(-\infty, 0)$ and decreasing from $\frac{1}{4}$ to 0 on $(0,+\infty)$. So, necessarily, $\lambda \in\left(0, \frac{1}{4}\right)$ and

$$
\varphi^{\prime-1}(\{\lambda(l)\})=\left\{z_{1}(l), z_{2}(l)\right\} \quad \text { with }-1<z_{1}(l)<0<z_{2}(l) .
$$

Then, let us remark that since $\varphi$ is increasing on $(-1,+\infty)$, one has

$$
\varphi\left(z_{2}\right)>\varphi\left(z_{1}\right) \text {. }
$$

Next, denote by $j$ the number of $a_{k}$ that are equal to $z_{1}$ (thus, $l-j a_{k}$ are equal to $z_{2}$ ). One has $j=j(l)$ and $1 \leq j \leq l-1$. Hence (5.10) becomes

$$
\begin{aligned}
\varphi_{h}^{* *}(0) & =h\left(j \varphi\left(z_{1}\right)+(l-j) \varphi\left(z_{2}\right)\right) \\
& \geq \varphi\left(z_{1}\right) \quad \text { by }(5.12) .
\end{aligned}
$$


Therefore, Theorem 1.1 implies that $\lim _{l \rightarrow+\infty} \varphi\left(z_{1}(l)\right)=0$, which leads to

$$
\lim _{l \rightarrow+\infty} z_{1}(l)=-1
$$

By (5.11) we have now $\lim _{l \rightarrow+\infty} \lambda(l)=0$, and then

$$
\lim _{l \rightarrow+\infty} z_{2}(l)=+\infty
$$

Consequently, from (5.13) and (5.14) we obtain

$$
\frac{l-j(l)}{j(l)}=\frac{-z_{1}(l)}{z_{2}(l)} \sim \frac{1}{z_{2}(l)} \quad \text { when } \quad l \rightarrow+\infty
$$

and also

$$
\frac{j(l)}{l}=\frac{z_{2}(l)}{z_{2}(l)-z_{1}(l)} \sim 1 \quad \text { when } \quad l \rightarrow+\infty
$$

Hence

$$
\begin{aligned}
\varphi_{h}^{* *}(0) & =\frac{j(l)}{l} \varphi\left(z_{1}(l)\right)+\frac{l-j(l)}{l} \varphi\left(z_{2}(l)\right) \\
& \geq \frac{l-j(l)}{j(l)} \cdot \frac{j(l)}{l} \varphi\left(z_{2}(l)\right) \quad \text { since } \varphi \geq 0 \\
& \sim \frac{\varphi\left(z_{2}(l)\right)}{z_{2}(l)} \quad \text { when } l \rightarrow+\infty
\end{aligned}
$$

So,

$$
\varphi_{h}^{* *}(0) \geq \frac{1}{2 \ln \left(z_{2}(l)\right)} \quad \text { when } l \rightarrow+\infty
$$

But $z_{2} \leq(l-j) z_{2}=-j z_{1} \leq l$, thus $\ln \left(z_{2}(l)\right) \leq \ln (l)$, and then (5.15) implies

$$
\varphi_{h}^{* *}(0) \geq \frac{1}{2 \ln \left(\frac{1}{h}\right)}
$$

for $h$ small enough. This proves that we cannot hope for an estimate of $\varphi_{h}^{* *}(0)$ in terms of $C h^{s}$.

Remark 5.4. The previous example allows us to show that, when epi $\left(\varphi^{* *}\right)$ contains a line or an extreme half-line, the estimate of Corollary 4.1 is not possible in general.

Indeed, consider $\Omega=(0,1)^{2}$ split into triangles of diameters $h$; see Fig. 1 .

Consider the function of $\mathbb{R}^{2}$ defined by

$$
\psi\left(\beta_{1}, \beta_{2}\right)=\varphi\left(\beta_{1}\right)
$$

where $\varphi$ is the function of the preceding remark.

One clearly has

$$
\psi^{* *}(0,0)=0
$$




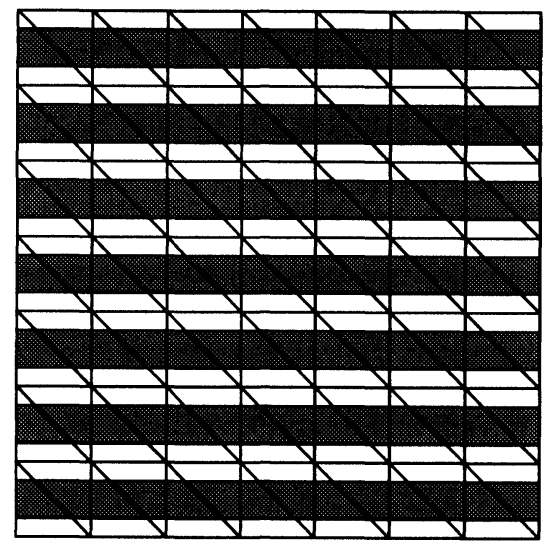

FIG. 1.

On the other hand,

$$
\begin{aligned}
\psi_{h}^{* *}(0,0) & =\operatorname{Inf}_{v \in V_{h}^{0}} \frac{1}{|\Omega|} \int_{\Omega} \psi(\nabla v(x, y)) d x d y \\
& =\operatorname{Inf}_{v \in V_{h}^{0}} \frac{1}{|\Omega|} \int_{\Omega} \varphi\left(\frac{\partial v}{\partial x}(x, y)\right) d x d y \\
& \geq \operatorname{Inf}_{v \in V_{h}^{0}} \frac{1}{|\Omega|} \int_{D} \varphi\left(\frac{\partial v}{\partial x}(x, y)\right) d x d y
\end{aligned}
$$

where $D$ denotes the doted region of the figure. Now on $D$, the function

$$
x \longmapsto v(x, y)
$$

is a piecewise affine function, vanishing at the endpoints $x=0, x=1$. Moreover, the mesh size associated to this function, i.e., the size of the intervals where $v(., y)$ is affine, is of the order of magnitude $h$. So, by the previous remark,

$$
\int_{D} \varphi\left(\frac{\partial v}{\partial x}(x, y)\right) d x d y \geq \frac{C}{\ln \left(\frac{1}{h}\right)}
$$

and thus

$$
\psi_{h}^{* *}(0,0)-\psi^{* *}(0,0)=\psi_{h}^{* *}(0,0) \geq \frac{C}{\ln \left(\frac{1}{h}\right)},
$$

which prevents Corollary 4.1 from holding.

One can argue the same way when $\operatorname{epi}\left(\varphi^{* *}\right)$ contains an extreme half-line.

\section{REFERENCES}

[1] B. BRIGHI, Sur quelques problèmes de Calcul des Variations et l'approximation de leur fonctionnelle relaxée, Ph.D. thesis, University of Metz, France, 1991. 
[2] B. BRIghi AND M. Chipot, Approximation in nonconvex problems, in Progress in Partial Differential Equations: Calculus of Variations, Applications, C. Bandle, J. Bemelmans, M. Chipot, M. Grüter, and J. Saint Jean Paulin, eds., Pitman Res. Notes Math. Ser. 267, Longman Scientific \& Technical, London, 1992.

[3] M. ChIPOT, Numerical analysis of oscillations in nonconvex problems, Numer. Math., 59 (1991), pp. 747-767.

[4] - Numerical analysis of oscillations in two well problems, in Progress in Partial Differential Equations: The Metz Surveys, M. Chipot and J. Saint Jean Paulin, eds., Pitman Res. Notes Math. Ser. 249, Longman Scientific \& Technical, London, 1991.

[5] M. ChIPOT AND C. Collins, Numerical approximation in variational problems with potential wells, SIAM J. Numer. Anal., 29 (1992), pp. 1002-1019.

[6] M. ChIPOT, C. Collins, AND D. KINDERLEHRER, Numerical analysis of oscillations in multiple well problems, in preparation.

[7] C. Collins, Computation and analysis of twinning in crystalline solids, Ph.D. thesis, University of Minnesota, Minneapolis, MN, 1990.

[8] C. Collins, D. Kinderlehrer, AND M. Luskin, Numerical approximation of the solution of a variational problem with a double well potential, SIAM J. Numer. Anal., 28 (1991), pp. 321-332.

[9] B. Dacorogna, Direct Methods in the Calculus of Variations, Applied Math. Sciences 78, Springer-Verlag, New York, 1989.

[10] I. Ekeland AND R. Temam, Analyse convexe et problèmes variationnels, Dunod, Paris, 1974.

[11] D. Gilbarg and N. S. Trudinger, Elliptic Partial Differential Equations of Second Order, Springer-Verlag, Berlin, 1985.

[12] P. Marcellini, Alcune osservazioni sull'esistenza del minimo di integrali del calcolo delle variazioni senza ipotesi di convessità, Rend. Mat., 13 (1980), pp. 171-281.

[13] E. MAscolo AND R. Schianchi, Existence theorems in the Calculus of Variations, J. Differential Equations, 67 (1987), pp. 185-198.

[14] P. A. RAVIART AND J. M. ThOMAs, Introduction à l'analyse numérique des équations aux dérivées partielles, Masson, Paris, 1988.

[15] R. T. Rockafellar, Convex Analysis, Princeton University Press, Princeton, NJ, 1970. 